\title{
Nuevos Descriptores en Ciencias de la Salud para clasificar y recuperar información sobre equidad
}

\author{
Ana Cristina Espíndola Campos ${ }^{1}$, Arthur Treuherz ${ }^{1}$, Renato Toshiyuki Murasaki', \\ Diego Gonzalez y Oscar J Mújica²
}

Forma de citar

Campos ACE, Treuherz A, Murasaki RT, Gonzalez D, Mújica OJ. Nuevos Descriptores en Ciencias de la Salud para clasificar y recuperar información sobre equidad. Rev Panam Salud Publica. 2020;44:e98. https://doi.org/10.26633/RPSP.2020.98

RESUMEN El vocabulario Descriptores en Ciencias de la Salud (DeCS) establece un lenguaje único y común que permite
la organización y facilita la búsqueda y recuperación de la literatura técnica y científica en salud disponible en
las fuentes de información de la Biblioteca Virtual en Salud. El DeCS, creado por el Centro Latinoamericano
y del Caribe de Información en Ciencias de la Salud (BIREME), un centro especializado de la Organización
Panamericana de la Salud/Organización Mundial de la Salud (OPS/OMS), es la traducción y la extensión del
vocabulario Medical Subject Headings (MeSH), mantenido por la National Library of Medicine de los Estados
Unidos. BIREME, en coordinación con expertos de América Latina y el Caribe, ha incluido en el DeCS los
temas de equidad, género, etnicidad y derechos humanos —-temas transversales en el marco programático
de la cooperación técnica de la OPS/OMS- para garantizar una mejor recuperación y uso de la información y
evidencia científica relacionadas a estos temas. El objetivo de este artículo es describir el método de revisión
terminológica del DeCS e informar los resultados obtenidos y los impactos de la ampliación terminológica en
el área de equidad, que comprendió la inclusión de 35 nuevos descriptores.

Palabras clave Equidad; sistemas de información; Medical Subject Headings; práctica clínica basada en la evidencia; acceso a la información.

El uso de vocabularios controlados establece un lenguaje único que permite la organización y facilita la búsqueda y recuperación de información, una vez que orienta su descripción e indización por medio de relaciones entre conceptos terminológicos de un área de conocimiento. El vocabulario estructurado y multilingüe Descriptores en Ciencias de la Salud (DeCS) (1) cumple este rol para la literatura técnica y científica en salud disponible en las principales fuentes de información de la Biblioteca Virtual en Salud (BVS), integrando la metodología LILACS (2).

El origen del DeCS se remonta a fines de los años 70, cuando el Centro Latinoamericano y del Caribe de Información en Ciencias de la Salud (BIREME), un centro especializado de la Organización Panamericana de la Salud/Organización Mundial de la Salud (OPS/OMS), comenzó a colaborar con la

\footnotetext{
1 Centro Latinoamericano y del Caribe de Información en Ciencias de la Salud, Organización Panamericana de la Salud. San Pablo, Brasil. $\square$ Ana Cristina Espíndola Campos, camposan@paho.org
}

National Library of Medicine (NLM) de los Estados Unidos en la traducción del vocabulario Medical Subject Headings (MeSH) al portugués y el español. Una vez identificada la necesidad de expandir la terminología ofrecida por la NLM, BIREME comenzó también a desarrollar y mantener, en tres idiomas, de manera integrada y compatible con el MeSH, las categorías para Ciencia y Salud, Homeopatía, Salud Pública y Vigilancia Sanitaria. La edición 2019 del DeCS comprende 33966 descriptores y calificadores; de ellos, 29431 se corresponden con el MeSH y 4 535 son exclusivos del DeCS.

Los retos relacionados con la organización y la recuperación de la información relevante frente al crecimiento exponencial de la información disponible son múltiples, y los vocabularios controlados son esenciales para superarlos. Durante las actualizaciones anuales del DeCS que siguen a las actualizaciones

\footnotetext{
2 Organización Panamericana de la Salud, Washington DC, Estados Unidos de América.
} 
anuales del MeSH y en otras ocasiones BIREME ha promovido estudios de evaluación terminológica para proponer a la NLM cambios en el MeSH, además de realimentar las propias categorías exclusivas del DeCS.

La alineación de BIREME con las actividades y programas de cooperación técnica de la OPS hizo evidente la necesidad de evaluar la cobertura del DeCS para las cuatro dimensiones que contextualizan transversalmente el abordaje de los determinantes sociales de la salud —equidad (3), género, etnicidad y derechos humanos. Estas dimensiones tornan explícito el compromiso por reducir las desigualdades en salud, reconocido por la OPS/OMS en su Plan Estratégico 2020-2025: La equidad, el corazón de la Salud (4). A fin de contribuir a la mejor organización, búsqueda y recuperación de información relacionada con estos temas transversales, BIREME ha desarrollado una metodología para evaluar la representatividad terminológica en el DeCS/MeSH (5) y proponer su extensión a partir del mapeo y análisis de vocabularios y documentos de referencia existentes.

Promover la equidad en salud está en el centro de la misión de la OPS y representa un principio fundamental para la acción política en salud (6). En los últimos años, la equidad en salud ha adquirido alta visibilidad y relevancia mundial, como se refleja en la expansión exponencial del tema -especialmente las desigualdades sociales en salud-en la literatura científica mundial y regional (6). En este contexto, maximizar la capacidad de recuperación específica de esta información es una prioridad. El objetivo de este artículo es describir el método de revisión terminológica del DeCS aplicado al tema de la equidad en salud y evaluar los impactos de la inclusión de 35 nuevos descriptores en la versión 2019 del DeCS.

\section{MATERIALES Y MÉTODOS}

El método de identificación y ampliación de la representación del área de equidad en el DeCS/MeSH siguió cinco etapas:

Etapa 1. Análisis de representatividad del término equidad en el DeCS/MeSH. La primera actividad consistió en identificar los términos existentes en el DeCS cuyo nombre incluyera la palabra equidad en los idiomas portugués, español e inglés. Este proceso fue realizado por la búsqueda booleana equidad OR equidade OR equity en los campos de descriptor, sinónimo e histórico de la base de datos DeCS/MeSH. Después de listar los términos encontrados, se eliminaron los términos no relacionados con la equidad o que tuvieran más de un significado y que, por lo tanto, ocasionarían la recuperación de documentos ajenos al tema. En segundo lugar, se identificaron los descriptores específicos de las ramas jerárquicas de aquellos encontrados anteriormente por medio de búsquedas en el índice jerárquico del DeCS/MeSH. Los términos encontrados fueron añadidos a los obtenidos en la primera actividad. Finalmente, se generaron expresiones de búsqueda para cada concepto DeCS/ $\mathrm{MeSH}$ encontrado en las actividades anteriores, incluyendo el descriptor y sus sinónimos en los tres idiomas, ejecutado en las fuentes de información de la BVS en su interfaz de búsqueda. Las expresiones de búsqueda siguieron el patrón "descriptor en inglés" OR "descriptor en español" OR "descriptor en portugués" $O R$ "sinónimo 1 en inglés" OR "sinónimo 1 en español" $O R$ "sinónimo 1 en portugués" $O R X$, donde $X$ es la expresión booleana OR de todos los sinónimos de un descriptor, en los tres idiomas. Cada expresión de búsqueda generada es parte de una sola expresión, combinándolas con el operador OR, de manera que se puedan recuperar los documentos relacionados en la BVS.

Etapa 2. Búsqueda por vocabularios relacionados a la equidad. Se buscó en Google vocabularios sobre equidad empleando la expresión de búsqueda (tesaur* OR thesaur* OR taxonom $^{*}$ OR vocabul* OR dicionário* OR dictionar ${ }^{*}$ OR diccionario* OR glossário* OR glosario* OR glossary) AND (Equidad* OR Equity). La selección de los vocabularios se basó en a) Orden en que aparece en el resultado de búsqueda; b) Tipo de institución asociada a la publicación del vocabulario (organizaciones internacionales, gobiernos, universidades, fundaciones, organizaciones no gubernamentales, etc.); c) Estructuración de los términos, con preferencia por taxonomías, tesauros y clasificaciones estructuradas jerárquicamente; d) Preferencia por sustantivos, con evitación de la jerga, aunque algunos adjetivos se incluyeron como términos alternativos de los respectivos sustantivos cuando eran términos consagrados por el uso en esa área; e) Selección de vocabularios en inglés, español y portugués, conforme al ámbito de trabajo de BIREME con el DeCS/ $\mathrm{MeSH}$; y f) Disponibilidad gratuita del texto completo de los documentos de referencia. A continuación, se seleccionaron los términos encontrados en los vocabularios identificados en la actividad anterior que estuvieran relacionados directamente con equidad. Finalmente, se tradujo, se agrupó conceptualmente y se seleccionó los términos preferidos, clasificándolos como descriptor o sinónimo DeCS/MeSH.

Etapa 3. Identificación de más descriptores y comparación cuantitativa de documentos recuperados. Se recuperaron documentos en la BVS a partir de la expresión de búsqueda resultante de la combinación de términos encontrados en las etapas 1 y 2, utilizando el operador OR, y se comparó las cantidades de documentos recuperados con el resultado de la búsqueda de la etapa 1 para identificar la representatividad de los términos de otros vocabularios en la BVS y cuantificar en qué medida su utilización ha expandido la capacidad de recuperación de documentos. Finalmente, se identificaron otros descriptores DeCS/MeSH utilizados en la indización de los documentos recuperados en la actividad anterior por medio del análisis del filtro "asunto principal", disponible en la interfaz de búsqueda de la BVS. Un análisis cualitativo de los resultados obtenidos mediante este filtro proporcionó otros descriptores pertinentes a la investigación más amplia sobre el tema.

Etapa 4. Propuesta de nuevos descriptores a la jerarquía existente. Se analizaron los nuevos conceptos identificados en las etapas anteriores y la posibilidad de incorporarlos a la jerarquía del DeCS/MeSH, obedeciendo a los códigos jerárquicos y a las especificaciones de alcance de los nuevos términos. Este trabajo se limitó a las categorías exclusivas del DeCS, manejadas por BIREME. Se sugirió al equipo de terminología de la NLM aquellos términos que también podrían ser incorporados en las categorías del MeSH.

Etapa 5. Revisión de la propuesta de extensión terminológica por expertos. Se sometió la propuesta de inclusión de nuevos descriptores a expertos en el área de estudio, inclusive profesionales a nivel nacional e internacional, a través de los Asesores Regionales de la OPS/OMS. El grupo de expertos evaluó la propuesta y envió a BIREME su opinión acerca de los 
nuevos descriptores, que fue considerada en el proceso final de actualización del DeCS/MeSH.

\section{RESULTADOSY DISCUSIÓN}

En la etapa 1 se identificaron 28 descriptores ya incluidos en el DeCS/MeSH asociados al tema de la equidad: Accesibilidad a los servicios de salud; Asignación de recursos para la atención de salud; Asistencia sanitaria culturalmente competente; Calidad de la atención de salud; Condiciones sociales; Derechos de la mujer; Desigualdades en la salud; Disparidades en atención de salud; Disparidades en el estado de salud; Educación de la población; Equidad en cobertura; Equidad en el acceso al agua; Equidad en el acceso; Equidad en la asignación de recursos; Equidad en salud; Equidad; Estrategia de salud familiar; Factores socioeconómicos; Género y salud; Indicadores de calidad de la atención de salud; Indicadores de salud; Inequidad social; Justicia social; Marginación social; Objetivos del desarrollo sostenible; Proyectos de inversión social; Salud global; y Sistemas de salud. A través de estos 28 descriptores del DeCS/MeSH se recuperaron 734444 documentos disponibles en la BVS en junio de 2017.

En la etapa 2 se identificaron a través de Google 14 fuentes de información (8-21) que resultaron en la selección de 139

CUADRO 1. Nuevos descriptores relacionados con la equidad

\begin{tabular}{|c|c|}
\hline Nuevos descriptores de equidad & Descriptor más amplio \\
\hline Cobertura universal de salud & Accesibilidad a los servicios de salud \\
\hline Capital social cognitivo & \\
\hline Capital social de redes & Capital social \\
\hline Capital social negativo & \\
\hline Efectos acumulativos de las desigualdades en salud & \\
\hline $\begin{array}{l}\text { Efectos contextuales de las desigualdades en salud } \\
\text { Efectos de vías de las desigualdades en salud }\end{array}$ & Disparidades en el estado de salud \\
\hline Efectos latentes de las desigualdades en salud & \\
\hline Equidad horizontal & \\
\hline $\begin{array}{l}\text { Equidad vertical } \\
\text { Estratificadores de equidad }\end{array}$ & Equidad en salud \\
\hline Políticas públicas antidiscriminación & \\
\hline Expectativa de salud & \\
\hline $\begin{array}{l}\text { Índice de desigualdad de la pendiente } \\
\text { Índice de disimilitud }\end{array}$ & Estado de salud \\
\hline Ecoepidemiología & Estudios ecológicos \\
\hline Epidemiología social & Estudios epidemiológicos \\
\hline Gradiente socioeconómico de salud & \\
\hline $\begin{array}{l}\text { Indicadores compuestos } \\
\text { Indicadores de desigualdad en salud }\end{array}$ & Factores socioeconómicos \\
\hline Cohesión social & Factores sociológicos \\
\hline Coeficiente de Gini & Indicadores de desarrollo \\
\hline $\begin{array}{l}\text { Indicadores trazadores } \\
\text { Índice de concentración }\end{array}$ & Indicadores de desigualdad en Salud \\
\hline Indicadores de enfermedades crónicas & \\
\hline $\begin{array}{l}\text { Indicadores de salud comunitaria } \\
\text { Índice de vulnerabilidad social }\end{array}$ & Indicadores de salud \\
\hline Observatorios de salud & Monitoreo de las desigualdades en salud \\
\hline Declaración de Alma-Ata & \\
\hline Desarrollo de la salud & \\
\hline Empoderamiento para la salud & Promoción de la salud \\
\hline Logros en salud & \\
\hline Monitoreo de la desigualdad en salud & \\
\hline Producto nacional bruto per cápita & Renta per cápita \\
\hline Riesgo atribuible a la población & Tasas, razones y proporciones \\
\hline
\end{tabular}


FIGURA 1. Distribución porcentual de los 35 nuevos descriptores y sinónimos relacionados con la equidad por categorías del DeCS/MeSH

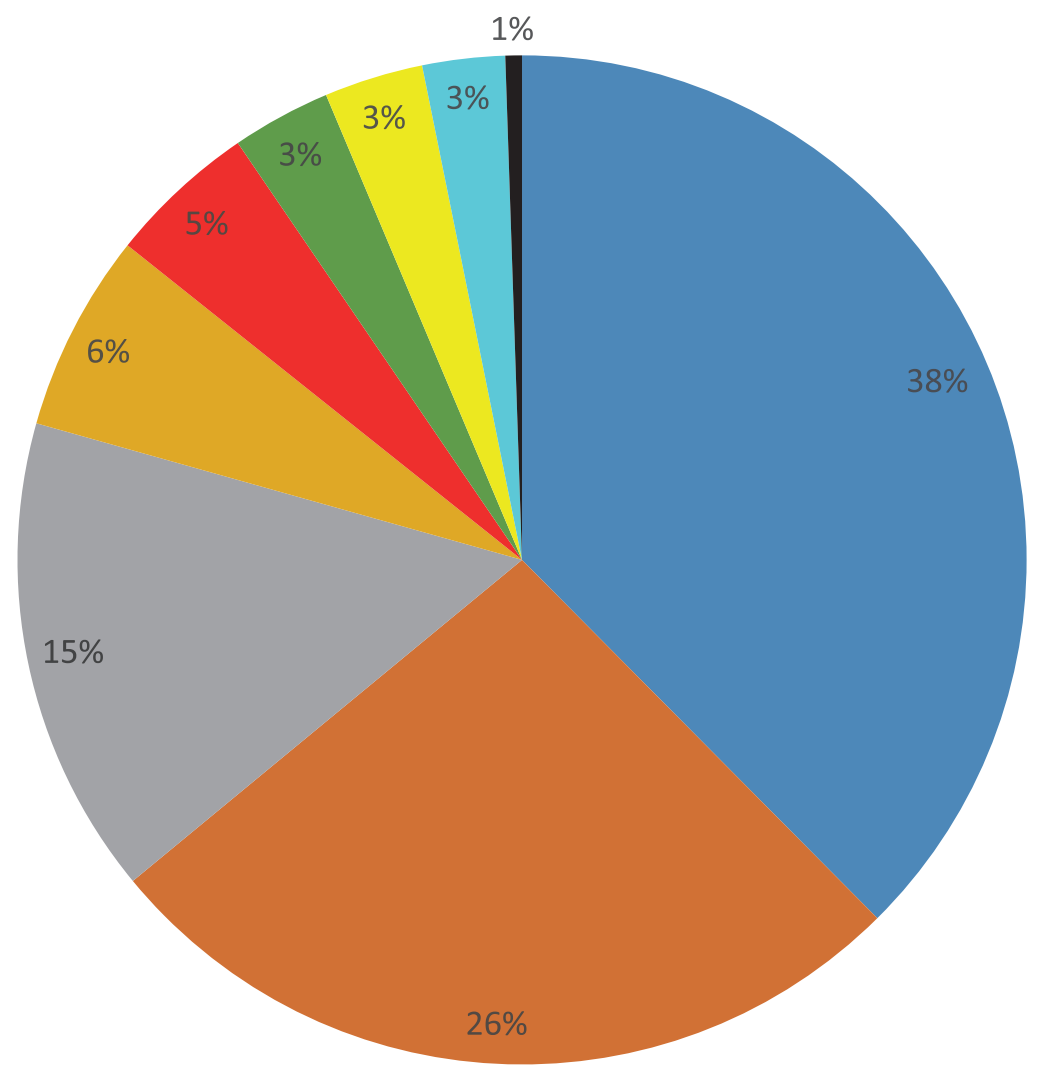

- Salud Publica

Atención de Salud

Antropología, Educación, Sociología y Fenómenos Sociales

Vigilancia Sanitaria

Disciplinas y Ocupaciones

- Ciencia y Salud

Psiquiatría y Psicología

Técnicas y Equipos Analíticos, Diagnósticos y Terapéuticos

Humanidades

términos, 91 de los cuales ya estaban representados en el DeCS/ $\mathrm{MeSH}$ o pudieron ser incluidos como sinónimos.

En la etapa 3 se recuperaron 748041 documentos a partir de la BVS y se identificaron, mediante el análisis del filtro "asunto principal", 12 descriptores más. Tras analizar los términos encontrados, se propuso la adición de 44 nuevos términos en la etapa 4.

Por último, en la etapa 5, los expertos eliminaron 11 descriptores y propusieron 6. De estos 6, se eliminaron aquellos poco específicos y los que ya existían en el DeCS. El resultado final fue la adición de 35 términos como nuevos descriptores en categorías exclusivas del DeCS (Cuadro 1). Cuando se consideran todos los nuevos descriptores y sinónimos relacionados con la equidad, se observa que la mayoría de ellos corresponden a la categoría de Salud Pública, lo que es esperable dado que es una categoría sobre la cual BIREME tiene injerencia (Figura 1).

Se añadieron como sinónimos de los nuevos descriptores propuestos o de los ya existentes otros 44 términos, inclusive en categorías del MeSH: Acción comunitaria para la salud; Ambiente político; Análisis de situación de salud; Años esperados de estudio; Ciudadanía en salud; Colaboración para la promoción de la salud; Desigualdades raciales en la salud; Desigualdades socio-espaciales en salud; Disparidades de género en salud; Disparidades espaciales en salud; Disparidades étnicas en salud; Disparidades financieras en salud; Enfermedad de declaración obligatoria; Entornos que apoyan la salud; Equidad de género en salud; Equidad en atención a la salud; Estratificación social; Evaluación de la promoción de la salud; Ganancia de salud; Gradiente social de salud; Habilidades para la vida; Habilidades personales; Indicador sensible al género; Indicadores de rendimiento del sistema de salud; Indicadores del estado de salud; Índice de desarrollo humano; Inequidad de las políticas económicas; Inequidad de las políticas económicas y sociales; Informes sobre la desigualdad en salud; Infraestructura para la promoción de la salud; Innovaciones; Inversión para la salud; Metas de salud; Objetivos de salud; Percepción de equidad; Política pública saludable; Políticas ecuánimes de salud; Responsabilidad social para la salud; Resultados de la promoción de la salud; Resultados de salud; Resultados intermedios de salud; Seguimiento de la desigualdad en salud; Soporte social; y Tiempo de escolarización.

Además de aumentar la cantidad de documentos recuperados en el portal la BVS, diversos términos propuestos en este estudio permiten recuperar documentos relevantes en MEDLINE, lo que sugiere que tienen potencial de convertirse en descriptores del MeSH. De hecho, los descriptores surgidos de este trabajo "Políticas públicas antidiscriminación" e "Indicadores de 
enfermedades crónicas", por ejemplo, se incluyeron después en el MeSH en el marco de la colaboración de BIREME con la NLM para la traducción del MeSH.

La inclusión de los nuevos descriptores permite que el tema de la equidad esté más ampliamente representado en el DeCS/ $\mathrm{MeSH}$. La dispersión de los conceptos por las ramas del árbol jerárquico del tesauro muestra su multidisciplinariedad o transversalidad. Los nuevos descriptores del tema equidad se concentran principalmente bajo el concepto de "Promoción de la salud". La indización de artículos y otros materiales científicos con los descriptores adecuados aumenta la especificidad de la recuperación de información, una prioridad para la promoción de la equidad en salud.
Contribución de los autores. ACEC, AT y RTM contribuyeron al desarrollo de la metodología, el análisis, la propuesta y ampliación terminológica del DeCS y la redacción del manuscrito. DG contribuyó con la revisión del manuscrito y OJM con la revisión de la propuesta de ampliación terminológica del DeCS y la revisión del manuscrito. Todos los autores revisaron y aprobaron la versión final para publicación.

Conflicto de intereses. Ninguno declarado.

Declaración. Las opiniones expresadas en este manuscrito son responsabilidad del autor y no reflejan necesariamente los criterios ni la política de la RPSP/PAJPH y / o de la OPS.

\section{REFERENCIAS}

1. DeCS [Internet]. Descriptores en Ciencias de la Salud [acceso en 15 feb 2020]. Disponible en: http://decs.bvsalud.org/E/homepagee. htm.

2. BIREME/OPS/OMS. Manual de Indexação de Documentos para a Base de Dados LILACS. [Internet] 2008. São Paulo. Disponible en: http://metodologia.lilacs.bvs.br/download/P/LILACS-4-Manual Indexacao-pt.pdf

3. Organización Panamericana de la Salud. 29a . Conferencia Sanitaria Panamericana. $69^{\mathrm{a}}$. Sesión del Comité Regional de la OMS para las Américas. Washington, DC: OPS; 2017. [acceso en 5 mar 2020]. Disponible en: http://iris.paho.org/xmlui/bitstream/ handle / $123456789 / 49169 /$ CSP296-spa.pdf? sequence=1\&is Allowed $=y$

4. Organización Panamericana de la Salud. Plan Estratégico de la Organización Panamericana de la Salud 2020-2025. 57 ${ }^{\circ}$. Consejo Directivo. $71^{\circ}$. Sesión del Comité Regional de la OMS para las Americas; Washington, DC: OPS; 2019. [acceso en 06 mar 2020]. Disponible en: http://iris.paho.org/xmlui/bitstream/handle/123456789/51599/ CD57-OD359-s.pdf? sequence $=2 \&$ isAllowed $=y$

5. BIREME/OPS/OMS. Equidad en salud, diseminación de información y la cooperación técnica de la OPS/OMS. Boletin $\mathrm{n}^{\circ}$. 19: 2018. São Paulo. [acceso en 27 feb 2020]. Disponible en: http:// boletin.bireme.org/2018/04/27/equidad-en-salud-diseminacionde-informacion-y-la-cooperacion-tecnica-de-la-opsoms /

6. Mújica OJ, Moreno CM. De la retórica a la acción: medir desigualdades en salud para "no dejar a nadie atrás". Rev Panam Salud Publica. 2019;43:e12. Disponible en: https://doi.org/10.26633/ RPSP.2019.12

7. Bouchard L, Albertini M, Batista R, de Montigny J. Research on health inequalities: A bibliometric analysis (1966-2014) [published correction appears in Soc Sci Med. 2015 Nov;145:140]. Soc Sci Med. 2015;141:100-108. Disponible en: https://doi.org/10.1016/j. socscimed.2015.07.022

8. Brasil. Ministério da Saúde. Análise de situação de saúde/ Ministério da Saúde, Universidade Federal de Goiás. Brasília, 2015. [acceso en 04 mar 2020]. Disponible en: http://bvsms.saude.gov.br/bvs/ publicacoes/asis_analise_situacao_saude_volume_1.pdf

9. Arcaya MC, Arcaya AL, Subramanian SV. Inequalities in Health: definitions, concepts and theories. Glob Health Action. 2015;8:27106. Disponible en: https:/ / doi.org/10.3402/gha.v8.27106

10. cdc.gov [Internet]. Sources for data on social determinants of health. Atlanta: Centers for Disease Control and Prevention; 2018 [acceso en 05 mar 2020]. Disponible en: https://www.cdc.gov/ socialdeterminants/data/index.htm

11. Fundação Osvaldo Cruz [Internet]. Glossário. Rio de Janeiro: Fundação Osvaldo Cruz; 2010 [acceso 4 mar 2020]. Disponible en: https://portal.fiocruz.br/noticia/glossario

12. Organización Mundial de la Salud [Internet]. Promoción de la Salud. Glosario. Subdirección General de Epidemiología, Promoción y Educación para la Salud; Ginebra: OMS; 1998 [acceso en 04 mar 2020]. Disponible en: https://apps.who.int/iris/bitstream/ handle/10665/67246/WHO_HPR_HEP_98.1_spa.pdf;jsessionid= 8E799EA9287DE257361816D165FF0001? sequence $=1$

13. Pan American Health Organization [Internet]. Portal for the Equity List and knowledge Network. Washington DC: PAHO; 2018 [acceso en 05 mar 2020]. Disponible en: http://equity.bvsalud.org/ glossary

14. Pan American Health Organization [Internet]. Determinantes e Desigualdades em Saúde; Washington DC: PAHO; 2012 [acceso en 04 mar 2020]. Salud en las Americas. Disponible en: https: / / www.paho. org/salud-en-las-americas-2012/index.php?option=com_content\& view $=$ article \&id=58:health-determinants-and-inequalities\& Itemid $=165 \&$ lang $=\mathrm{pt}$

15. World Health Organization [Internet]. Gender, equity and human rights. Glossary of terms and tools. Geneva: WHO; 2011 [acceso em 06 mar 2020]. Disponible en: https://www.who.int/ gender-equity-rights/knowledge/glossary/en/

16. World Health Organization [Internet]. A Glossary of Terms for Community Health Care and Services for Older Persons. Ageing and Health Technical Report. Geneva: WHO Centre for Health Development; 2004 [acceso en 05 mar 2020]. Disponible en: https://apps. who.int/iris/bitstream/handle/10665/68896/WHO_WKC_Tech. Ser._04.2.pdf?sequence $=1 \&$ isAllowed $=y$

17. World Health Organization. Handbook on Health inequality monitoring: with a special focus on low- and middle-income countries. Geneva: WHO; 2004. [acceso en 05 mar 2020]. Disponible en: https://apps.who.int/iris/bitstream/handle/10665/85345/97892 41548632_eng.pdf?sequence $=1$

18. World Health Organization [Internet]. Health Impact Assessment (HIA). Glossary of Terms used [acceso en 18 ene 2020]. Geneva: WHO; 2018. Disponible en: https:/ / www.who.int/publications/m/item/ glossary-of-terms-used-for-health-impact-assessment-hia

19. World Health Organization [Internet]. Health Systems: Equity [acceso en 05 feb 2020]. Disponible en: https://www.who.int/ healthsystems/topics/equity/en/

20. Observatório sobre Iniquidades em Saúde [Internet]. Rio de Janeiro: Portal DSS Brasil; 2012 Mar 27. [acceso en 05 mar 2020]. Disponible en: http://dssbr.org/site/2012/03/observatorio-sobreiniquidades-em-saude/

21. Lobo A, Mateus S. Validity and Reliability of an Equity in Health Care Scale improvement. SAGE Open. 2013 Oct 7. Disponible en: https://doi.org/10.1177/2158244013506716

Manuscrito recibido el 3 de abril de 2020. Aceptado para su publicación, tras revisión, el 26 de mayo de 2020. 


\section{New Health Science Descriptors to classify and retrieve information on equity}

ABSTRACT The Health Sciences Descriptors (DeCS) vocabulary establishes a unique and common language that allows the organization and facilitates the search and retrieval of technical and scientific literature on health available in the information sources of the Virtual Health Library. The DeCS, created by the Latin American and Caribbean Center on Health Sciences Information (BIREME), a specialized center of the Pan American Health Organization/World Health Organization (PAHO/WHO), is the translation and extension of the Medical Subject Headings (MeSH) vocabulary, maintained by the United States National Library of Medicine. BIREME, in coordination with experts from Latin America and the Caribbean, has included in the DeCS the topics of equity, gender, ethnicity and human rights - cross-cutting themes in the programmatic framework of $\mathrm{PAHO} / \mathrm{WHO}$ technical cooperation - to ensure better retrieval and use of scientific information and evidence related to these topics. The objective of this article is to describe the methodology used during the terminology review of the DeCS and to report the results obtained and the impacts of the terminology expansion in the field of equity, which included the inclusion of 35 new descriptors.

Keywords Equity; information systems; Medical Subject Headings; evidence-based practice; access to information. 